\title{
Diferencias según sexo en el tratamiento y la evolución de los pacientes afectados de síndrome coronario agudo
}

\author{
Gender differences in the treatment and outcome of patients with \\ acute coronary syndrome
}

\author{
M. Ferraz-Torres ${ }^{1}$, T. Belzunegui-Otano ${ }^{2}$, B. Marín-Fernández ${ }^{3}$, Ó. Martínez-García ${ }^{4}$, \\ X. Jiménez Fábregas ${ }^{5}$
}

\section{RESUMEN}

Fundamento. La perspectiva de género en salud nos alerta de la diferente prevalencia, incidencia, evolución y letalidad de las patologías coronarias agudas según sexo. Este estudio pretende conocer la diferencia en el tratamiento y la evolución de los pacientes afectos de Síndrome Coronario Agudo (SCA) según sexos en Navarra.

Métodos. Se analizaron 35 variables de 130 usuarios que acudieron consecutivamente al servicio de Urgencias (SU) del Complejo Hospitalario de Navarra (CHN) con patología coronaria aguda desde enero hasta abril de 2012. La variable dependiente fue el sexo y las independientes los tiempos, tratamientos y evolución final del proceso.

Resultados. Un 74,6\% de la muestra fueron varones con una edad media de 67 años, inferior a los 72 años de la muestra femenina ( $p=0,043)$. Se obtuvo una mediana de 3 factores de riesgo cardiovascular (FRC) en los hombres y de dos en las mujeres $(\mathrm{p}=0,026)$. El tiempo de demora generado por los pacientes fue de 161 minutos en varones vs 266 minutos en féminas $(\mathrm{p}=0,006)$. El tratamiento llevado a cabo mediante revascularización por angioplastia primaria (AP) o fibrinolisis se realizó en un $71,6 \%$ de los hombres y un $41,2 \%$ de las mujeres $(\mathrm{p}=0,002)$. Se registró un $5,9 \%$ de muertes en mujeres, sin hallarse casos de fallecimiento en varones $(\mathrm{p}=0,017)$.

Conclusiones. En Navarra, los procesos coronarios siguen siendo una patología de predominio masculino pero de mayor gravedad en mujeres. El tratamiento se realizó de forma distinta según sexo. Se observó un mayor retraso en la solicitud de atención sanitaria en las mujeres así como la presencia de alta voluntaria en ellas, lo que puede influir en la peor evolución de las mismas.

Palabras clave. Síndrome coronario agudo. Sexo. Tratamiento. Tiempos de respuesta. Causas.
1. DUE. Servicio de Urgencias. Complejo Hospitalario de Navarra. Pamplona.

2. Médico adjunto. Servicio de Urgencias. Complejo Hospitalario de Navarra. Pamplona.

3. Doctora en enfermería. Universidad Pública de Navarra. Pamplona.

4. Médico adjunto. Servicio de Anestesiología, Reanimación y Terapéutica del Dolor. Complejo Hospitalario de Navarra.

5. Jefe clínico. Servicio de Emergencias Médicas de Barcelona.

\begin{abstract}
Background. Gender-based approaches have revealed the differing prevalence, incidence, progression and mortality of acute coronary disease by sex. This study aims to determine the difference by sex in the treatment and outcomes of patients with acute coronary syndrome (ACS) in Navarre.
\end{abstract}

Methods. Thirty-five variables were analysed from 130 users with acute coronary disease who attended the Navarre Hospital (CHN) emergency department consecutively from January to April 2012. The dependent variable was sex and independent variables were time, treatments and final outcome of the process.

Results. Males accounted for $74.6 \%$ of the sample, with a mean age of 67 , which was less than the mean age of 72 for the female patients $(\mathrm{p}=0.043)$. The median for cardiovascular risk factors was three in men and two in women $(\mathrm{p}=0.026)$. The patient delay in seeking health care was 161 minutes in men compared to 266 minutes in women $(\mathrm{p}=0.006)$. Treatment via revascularization by primary angioplasty or fibrinolysis was performed in $71.6 \%$ of men and $41.2 \%$ of women ( $p=0.002$ ). A $5.9 \%$ death rate was registered for women, with no deaths among the men $(\mathrm{p}=0.017)$.

Conclusions. In Navarre, acute coronary syndrome remains more prevalent among men yet more severe in women. Treatment differs according to gender. Greater delay in seeking health care is observed among women, as is self-discharge from hospital, which may contribute to their less favourable outcomes.

Key words. Acute coronary syndrome. Sex. Treatment. Response times. Causes.

\section{Correspondencia: \\ M. Ferraz Torres}

Servicio de Urgencias

Complejo Hospitalario de Navarra

Irunlarrea, 3

31008 Pamplona

Recepción: 2 de marzo de 2014

Aceptación provisional: 23 de abril de 2014

Aceptación definitiva: 10 de junio de 2014 


\section{INTRODUCCIÓN}

El estudio de las desigualdades según género en el ámbito sanitario ha observado la presencia de diferencias biológicas (fisiopatológicas, hormonales etc.) que influyen en la salud y la enfermedad de las personas según sexo. La perspectiva de género en salud nos alerta de la diferencia según sexo en la prevalencia, incidencia, evolución y letalidad de las patologías coronarias agudas.

Uno de los pioneros en la investigación de las desigualdades según sexos fue el estudio Framingham ${ }^{1}$, el cual demostró que el riesgo de padecer una enfermedad coronaria es mayor para las mujeres que presentan un mismo nivel de factores de riesgo que los hombres.

La perspectiva actual nos confirma que a pesar de los avances obtenidos en la prevención y el tratamiento de estas patologías, en las mujeres se produce una mayor mortalidad precoz tras un infarto agudo de miocardio (IAM) que en los hombres ${ }^{2}$. Como posibles causas a esta peor evolución en las mujeres se ha mencionado la desigualdad en los cuidados médicos proporcionada para los hombres y para las mujeres, sin llegar a analizar la actuación llevada a cabo por el propio usuario ${ }^{3-5}$.

El estudio de la actuación sanitaria y el tratamiento aplicado según sexos en el servicio de Urgencias (SU) mediante la administración de beta-bloqueantes $(\mathrm{Bb})$, antiagregación, nitratos o inhibidores de la angiotensina, ha detectado una menor frecuencia en su administración en las mujeres, tanto en la fase aguda del proceso coronario como al alta ${ }^{6-9,10}$. La realización de pruebas diagnósticas como la angiografía coronaria o la intervención terapéutica de revascularización mediante la fibrinolisis o la angioplastia primaria (AP) también se ha visto influenciada por el sexo ${ }^{9,11,12}$. La mayor dificultad diagnóstica del IAM en las mujeres, así como la mayor longevidad de las féminas afectadas con estas patologías son elementos expresados como justificante a la menor administración de estos tratamientos ${ }^{4,11-15}$.

Esta diferencia de tratamiento recibido según sexos, junto con variables como la edad, el sexo, los factores de riesgo cardiovascular, los antecedentes coronarios, la localización del IAM y el tipo de hospital o área de ingreso figuran como aspectos que repercuten en la evolución del paciente, tanto a nivel de mortalidad temprana como tardía tras un proceso coronario ${ }^{4,5,13-15}$.

El objetivo de este estudio fue conocer las diferencias en el tratamiento y la evolución de los pacientes afectados de procesos coronarios según sexo en Navarra.

\section{MÉTODOS}

Se realizó un estudio analítico de carácter observacional prospectivo, mediante el estudio de pacientes atendidos en el SU del CHN A (hospital terciario de referencia para las patologías coronarias en Navarra), diagnosticados como SCA, desde el 1 de enero hasta de 30 de abril de 2012. Se recogieron todos los casos que tras la valoración inicial en el SU y en base a los hallazgos del electrocardiograma (ECG) fueron diagnosticados como SCA con elevación del ST (SCACEST) y SCA sin elevación del ST (SCASEST).

Se objetivó la necesidad de obtener una muestra de 130 pacientes para detectar un riesgo mínimo de 1,5 , mediante la aproximación de Poisson, aceptando un riesgo alfa de 0,05 , un riesgo beta inferior al 0,2 en un contraste bilateral y una tasa de pérdidas del $5 \%$.

Se recogieron 35 variables, distribuidas en datos demográficos, anamnesis, pruebas diagnósticas, tratamiento, tiempos $\mathrm{y}$ evolución.

Se efectuó una valoración de los tiempos mediante diferenciación de los mismos en t1 o tiempo-paciente como el tiempo transcurrido desde el comienzo de la sintomatología hasta la solicitud de asistencia sanitaria (tanto mediante llamada al 112 SOS-Navarra como la asistencia al centro de atención primaria o directamente al SUH del hospital); el t2 o tiempo de atención pre-hospitalaria como el tiempo que transcurre desde la atención del equipo sanitario de SOS-Navarra o el centro de atención primaria hasta la llegada del usuario al hospital; el t3 o tiempo triaje como 
el tiempo que transcurre desde el triaje del paciente hasta el comienzo de la asistencia sanitaria mediante la realización del electrocardiograma (ECG)), el t4 o tiempo puerta-aguja como el tiempo que abarca desde la realización del ECG hasta su tratamiento mediante fibrinolisis, el t5 o tiempobalón como el tiempo que abarca desde la realización del ECG hasta la realización de la AP y el t6 o tiempo de revascularización para los procesos tratados con cirugía de revascularización.

Para realizar el análisis estadístico se utilizó el programa SPSS $21 \circledR$. Se consideró estadísticamente significativo una $\mathrm{p}<0,05$.

Para responder a los objetivos del estudio se realizó en primer lugar la descripción de la frecuencia de todas las variables. Para las cuantitativas se estimaron como medida de tendencia central la media aritmética y como medida de dispersión la desviación estándar; para las variables cualitativas se estimaron proporciones como la frecuencia absoluta, frecuencia relativa y porcentajes. Posteriormente se llevó a cabo un análisis bivariado para establecer la posible existencia de diferencias estadísticamente significativas, siendo entre variables cualitativas la prueba de la Ji cuadrado y para el análisis de las variables cualitativas y cuantitativas con una distribución normal las pruebas $\mathrm{t}$ de student y ANOVA (previa comprobación de la homogeneidad de las varianzas mediante la prueba de Levene).

\section{RESULTADOS}

Un $74,6 \%$ de la muestra eran varones con una edad media de 67 años y las mujeres presentaron una edad media de 72 años $(\mathrm{p}=0,043)$. Se obtuvo una mediana de 3 factores de riesgo cardiovascular (FRC) en los varones y de dos en las mujeres $(\mathrm{p}=0,026)$ siendo el más frecuente en ellos el tabaco $(78,1 \%)$ seguido de la dislipemia $(69,8 \%)$ y la hipertensión arterial (HTA) (61,5\%). En las mujeres el FRC más frecuente resultó ser la HTA $(73,5 \%)$, seguida de la dislipemia $(52,9 \%)$ y el tabaco $(35,3 \%)$, siendo esta última la única significativa según sexos $(p<0,001)$.
El $94,1 \%$ de los hombres y el $91,1 \%$ de las mujeres $(p=0,451)$ reflejaron el inicio de la sintomatología como un dolor sugestivo de SCA (dolor torácico irradiado a brazo izquierdo o a ángulo mandibular, de tipo opresivo y asociado a síntomas vegetativos como sudoración profusa, nauseas o vómitos).

La creatin-kinasa miocárdica (CK-MB) presentó un nivel inicial diferente según sexos $(13,6 u i / l$ en hombres vs 19,9 ui/l en mujeres, $\mathrm{p}=0,092)$ así como la troponina $\mathrm{T}(\mathrm{TnT})$ $(0,7 \mathrm{ng} / \mathrm{ml}$ vs $3,2 \mathrm{ng} / \mathrm{ml}, \mathrm{p}=0,403)$ siendo los valores tras la seriación enzimática, pasadas 6 horas del inicio de la sintomatología, superior en los varones tanto para la CK-MB (75ui/l vs 20,4ui/l, p=0,058) como para la TnT $(15,5 \mathrm{ng} / \mathrm{ml}$ vs $5,6 \mathrm{ng} / \mathrm{ml}, \mathrm{p}=0,144)$.

Un $59,5 \%$ de los varones y un $43,5 \%$ de las mujeres presentaron algún tipo de lesión coronaria en la prueba de imagen ( $\mathrm{p}=0,358)$. Un $44,8 \%$ de los varones y un $41,2 \%$ de las féminas presentaron una alteración significativa de lesión coronaria aguda en el ECG ( $p=0,715)$.

La gravedad del SCACEST y del SCASEST se midió mediante la Escala de Riesgo TIMI, observándose una media de 3,68 en hombres y de 3,45 en mujeres $(p=0,278)$.

El estudio de los tiempos indicó una media para t1 de 161 min en varones vs 266 min en féminas $(\mathrm{p}=0,006), \mathrm{t} 4$ (70 min vs 181 min, $\mathrm{p}<0,001)$ y para t 5 de 45 min en hombres vs 166 min en mujeres $(\mathrm{p}<0,001)$. La media para $\mathrm{t} 2$ (48 $\mathrm{min}$ vs $47 \mathrm{~min}, \mathrm{p}=0,948)$, para $\mathrm{t} 3(11 \mathrm{~min}$ vs $14 \mathrm{~min}, \mathrm{p}=0,059)$ y $\mathrm{t} 6(1,4$ días vs 1,8 días, $\mathrm{p}=0,333$ ) fueron similares según sexos (Tabla 1).

Se registró la administración de antiagregación en un $94,1 \%$ de los varones y en un $91,1 \%$ de las mujeres ( $p=0,451)$, Bb en un $43,8 \%$ y un $44,1 \%$ de las mujeres ( $p=0,970)$, inhibidores de la angiotensina en un $47,9 \%$ y un $26,5 \%$ de las mujeres $(\mathrm{p}=0,03)$ y nitratos en un $94,8 \%$ de los hombres y un $91,2 \%$ de las mujeres $(\mathrm{p}=0,45)$ (Tabla 2). El tratamiento de revascularización llevado a cabo mediante AP o fibrinolisis se dio en un $71,6 \%$ de los hombres y un $41,2 \%$ de las mujeres $(\mathrm{p}=0,002)$ y la cirugía de revascularización coronaria o bypass en un 10,1\% de los varones y un $2,9 \%$ de las mujeres $(\mathrm{p}=0,040)$. 
Tabla 1. Comparación por sexo de las variables epidemiológicas, pruebas diagnósticas y tiempos de respuesta

\begin{tabular}{|c|c|c|c|}
\hline Variables & & Total & \\
\hline Variables epidemiológicas & $H(n=97)$ & $M(n=33)$ & Sig. ${ }^{(*)}$ \\
\hline Edad & $66,87 \pm 12,12$ & $72,42 \pm 16,50$ & 0,043 \\
\hline \multicolumn{4}{|l|}{ Factores de riesgo } \\
\hline A. Crónica & $30(31,3)$ & $11(32,4)$ & 0,533 \\
\hline Obesidad & $23(24)$ & $3(11,5)$ & 0,058 \\
\hline Tabaco & $75(78,1)$ & $12(35,3)$ & $<0,001$ \\
\hline Drogas & $3(3,1)$ & 0 & 0,297 \\
\hline Hipertensión & $59(61,5)$ & $25(73,5)$ & 0,206 \\
\hline Dislipemia & $67(69,8)$ & $18(52,9)$ & 0,076 \\
\hline Claudicación intermitente & $11(11,5)$ & $2(5,9)$ & 0,352 \\
\hline Diabetes mellitus & $21(21,9)$ & $11(32,4)$ & 0,223 \\
\hline Total F. Riesgo & $2,7 \pm 1,19$ & $2 \pm 1,1$ & 0,026 \\
\hline \multicolumn{4}{|l|}{ Anamnesis } \\
\hline Frecuencia Cardiaca (lpm) & $71 \pm 17,73$ & $73 \pm 20,18$ & 0,756 \\
\hline Tensión arterial sistólica (mmHg.) & $134 \pm 25,84$ & $139 \pm 31,84$ & 0,37 \\
\hline Fracción Eyección Ventricular (\%) & $52 \pm 10,93$ & $52 \pm 11,79$ & 0,807 \\
\hline \multicolumn{4}{|l|}{ Pruebas diagnósticas } \\
\hline Hemoglobina (g/dl) & $14,53 \pm 1,93$ & $12,85 \pm 1,65$ & $<0,001$ \\
\hline Creatinina $(\mathrm{g} / \mathrm{dl})$ & $1 \pm 0,4$ & $1 \pm 0,4$ & 0,279 \\
\hline Troponina T inicial $(\mu \mathrm{g} / \mathrm{l})$ & $0,7 \pm 2,59$ & $3,2 \pm 16,44$ & 0,403 \\
\hline Troponina T final $(\mu \mathrm{g} / \mathrm{l})$ & $15,5 \pm 29,19$ & $5,6 \pm 18,78$ & 0,144 \\
\hline Creatin-quinasaMB inicial (ui/l) & $13,6 \pm 17,09$ & $19,9 \pm 67,1$ & 0,092 \\
\hline Creatin-quinasa MB final (ui/l) & $75 \pm 15,06$ & $20,4 \pm 45,6$ & 0,058 \\
\hline Gravedad inicial (1-5) & $2,17 \pm 0,721$ & $2,38 \pm 0,739$ & 0,139 \\
\hline Lesión coronaria & $50(59,5)$ & $10(43,5)$ & 0,358 \\
\hline Alt. electrocardigrama & $43(44,8)$ & $14(41,2)$ & 0,715 \\
\hline Puntuación TIMI & $3,68 \pm 1,38$ & $3,45 \pm 1,69$ & 0,278 \\
\hline \multicolumn{4}{|l|}{ Tiempos } \\
\hline $\mathrm{t} 1$ (minutos) & $161 \pm 248,18$ & $266 \pm 363,47$ & 0,006 \\
\hline t2 (minutos) & $48 \pm 28,76$ & $47 \pm 23,98$ & 0,948 \\
\hline t3 (minutos) & $10,9 \pm 1,85$ & $13,9 \pm 8,34$ & 0,059 \\
\hline t4 (minutos) & $70 \pm 25,71$ & $181 \pm 101,12$ & $<0,001$ \\
\hline t5 (minutos) & $45 \pm 17,42$ & $166 \pm 101,08$ & $<0,001$ \\
\hline t6 (días) & $1,4 \pm 0,68$ & $1,8 \pm 0,96$ & 0.333 \\
\hline
\end{tabular}

Total $(\mathrm{n}=130)$

* Todas las comparaciones de frecuencias realizadas mediante el test Chi-cuadrado o test de Fisher, según correspondiera. Las comparaciones de medias mediante el test T-Student. Las comparaciones de medianas (Total Factores de Riesgo mediante test Chi-cuadrado de tendencias en proporciones). Los datos se expresan en n (\%) o media \pm desviación estándar excepto el número total de factores de riesgo que se expresa en mediana (rango). 
Tabla 2. Comparación por sexo de los tratamientos y evolución

\begin{tabular}{|c|c|c|c|}
\hline \multirow[t]{2}{*}{ Tratamiento } & \multicolumn{3}{|c|}{ Total } \\
\hline & $H(n=97)$ & $M(n=33)$ & $\operatorname{Sig}^{(*)}$ \\
\hline Antiagregación & $90(94,1)$ & $30(91,1)$ & 0,451 \\
\hline Nitratos & $91(94,8)$ & $31(91,2)$ & 0,450 \\
\hline Beta bloqueantes & $42(43,8)$ & $15(44,1)$ & 0,970 \\
\hline Inhibidores de angiotensina & $46(47,9)$ & $9(26,5)$ & 0,030 \\
\hline Angioplastia primaria/fibrinolisis & $68(71,6)$ & $14(41,2)$ & 0,002 \\
\hline Cirugía revascularización & $8(10,1)$ & $1(2,9)$ & 0,040 \\
\hline Cardioversión eléctrica & $1(1)$ & 0 & 0,550 \\
\hline Desfibrilación/RCP & 0 & $1(2,9)$ & 0,092 \\
\hline \multicolumn{4}{|l|}{ Evolución } \\
\hline Alta hospitalaria & $17(17,7))$ & $8(23,5)$ & 0,114 \\
\hline Hospitalización & $10(11,5)$ & $8(23,5)$ & \\
\hline UCC-UCI & $68(70,8)$ & $18(52,9)$ & \\
\hline Alta voluntaria & 0 & $1(2,9)$ & 0,027 \\
\hline Fallecimiento & 0 & $2(5,9)$ & 0,017 \\
\hline \multicolumn{4}{|l|}{ Total $(n=130)$} \\
\hline \multicolumn{4}{|c|}{$\begin{array}{l}\text { Todas las comparaciones de frecuencias realizadas mediante el test Chi-cuadrado o test de } \\
\text { Fisher, según correspondiera. Las comparaciones de medias mediante el test T-Student. Las } \\
\text { comparaciones de medianas (Total F Riesgo mediante test Chi-cuadrado de tendencias en } \\
\text { proporciones. }\end{array}$} \\
\hline
\end{tabular}

El estudio de la evolución final observó que un $11,55 \%$ de los varones y un $23,5 \%$ de las mujeres fueron hospitalizados en planta de cardiología ( $\mathrm{p}=0,114)$ y un $70,8 \%$ de los hombres y un $52,9 \%$ de las mujeres fueron ingresados en la Unidad de Cuidados Coronarios (UCC) o en Unidad de Cuidados Intensivos (UCI) $(\mathrm{p}=0,114)$. No se obtuvo ningún caso de alta voluntaria en varones pero sí se observó un caso en las mujeres $(\mathrm{p}=0,027)$.

Finalmente, se obtuvo un 5,9\% de fallecimientos en mujeres, sin registrarse casos en varones $(\mathrm{p}=0,017)$.

\section{DISCUSIÓN}

Se han encontrado diferencias en la aplicación del tratamiento de revascularización entre hombres y mujeres. Igualmen- te, se observaron diferencias en la actuación de los pacientes según sexo.

El análisis de las características demográficas en los pacientes afectados con patología coronaria aguda (SCACEST y SCASEST) según sexos, reflejó que las mujeres presentan una media de edad superior a la de los varones, diferencia significativa que confirma los resultados hallados en otros estudios $^{9,11,12}$.

A pesar de encontrar una mayor pluripatología en hombres, lo que podría indicar un perfil de riesgo cardiovascular más desfavorable para los varones, no se registraron diferencias en el estudio de la gravedad del proceso medido mediante la Escala de Riesgo TIMI ${ }^{9,11}$ según sexos. Tampoco se hallaron diferencias significativas en la gravedad del proceso observado mediante la coronariografía. Sin embargo, esto contra- 
dice la mayor incidencia de fallecimientos observada en las mujeres de este estudio, demostrándose la peor evolución del proceso en ellas ${ }^{23,6-8}$.

Este hecho puede estar influenciado por la mayor media de edad observada en las féminas ${ }^{6-8}$.

La menor aplicación de tratamiento de revascularización (fibrinolisis, $\mathrm{AP} \mathrm{o}$ bypass) registrado en ellas, junto con la mayor media de edad, actúan como factores lesivos para la peor evolución de las mujeres $^{8,9,11,13}$. El estudio de los tiempos, nos ha permitido observar un mayor retraso en solicitar la asistencia sanitaria por parte de las mujeres ${ }^{4-6,10}$ siendo de casi dos horas más, diferencia estadísticamente significativa ${ }^{3,15}$ que puede actuar como factor nocivo en la evolución de ellas.

La evolución hospitalaria examinada también es diferente según sexos, produciéndose mayor número de ingresos en la UCC o UCI en hombres afectos con SCA que en mujeres, sin llegar a tener una diferencia significativa estadísticamente ${ }^{6}$.

El estudio de la presencia de casos de alta voluntaria por parte de las mujeres, aspecto no estudiado en otras investigaciones nos ha permitido conocer la presencia de una diferencia significativa según sexos.

Nuestro estudio se llevó a cabo en un periodo corto de tiempo, por lo que sería conveniente ampliarlo para ver si estas diferencias según sexos se mantienen. Cabe citar que se trata de un estudio realizado en un único hospital terciario, que aun siendo hospital de referencia para la patología coronaria en Navarra, los resultados no son extrapolables a otras poblaciones.

En conclusión, la peor evolución observada en las mujeres afectadas con un SCA puede deberse a factores analizados en nuestro estudio, como la menor aplicación del tratamiento de revascularización en ellas y el mayor retraso generado por las mujeres en acudir al SU o solicitar la asistencia sanitaria.

Se presenta una diferencia significativa en la actuación profesional según sexos no justificable a la dificultad diagnóstica del proceso en las mujeres ni a una diferencia de gravedad del proceso según sexos.

\section{BIBLIOGRAFÍA}

1. LeRner DS, KANNEL WB. Patterns of coronary heart disease morbidity and mortality in the sexes: a 26-year follow-up of the Framingham population. Am Heart J 1986; 111: 383-390.

2. Marrugat J, Sala J, Aboal J. Epidemiología de las enfermedades cardiovasculares en la mujer. Rev Españ Cardiol 2006; 59: 264-274.

3. Carbajosa J, Llorens P, Diéguez S, Carratalá JM, Díaz J, Martínez E et al. Influencia del sexo del paciente en el manejo del síndrome coronario agudo con elevación del ST en los servicios de urgencias. Emergenias 2011; 23: 87-92.

4. Ferraz-Torres M, Belzunegui-Otano T, MarínFernández B, Martínez-García O, Azcona-Ciriza L, JiMÉNEZ-FÁBREGAS X. Percepción y actuación de los pacientes con patología coronaria aguda en la fase prehospitalaria. Met de enf 2014; 17: 6-11.

5. Bolívar-Muñoz J, Martínez-Cassinello R, MateoRodríguez I, Torres-Ruiz JM, Pascual-Martínez N, Rosell-Ortiz F et al. Actuación de los pacientes ante un síndrome coronario agudo: diferencias desde una perspectiva de género. Emergencias 2013; 25: 23-30.

6. Riesgo A, Miró Ó, López E, SÁnchez M. Comparación del manejo del infarto de miocardio sin elevación del ST durante la asistencia urgente en función del sexo del paciente. Rev Esp Cardiol 2011; 64: 1060-1064.

7. Moreno C, Galache JG, Sánchez-Rubio J, Calvo I, Ferrer MC, Placer LJ. Tratamiento de revascularización en fase aguda del infarto de miocardio con elevación del segmento ST en mujeres ancianas: eficacia en la reducción de la mortalidad. Med Clin 2010; 134: 333339 .

8. Bueno H, Bardají A, Fernández-Ortoz A, Marrugat J, Martí H, Heras M. Manejo del síndrome coronario agudo sin elevación del segmento ST en España. Estudio DESCARTES (Descripción del Estado de los Síndromes Coronarios Agudos en un Registro Temporal Español). Rev Esp Cardiol 2005; 58: 244-252.

9. Arós F, Cuñat J, Loma-Osorio A, Torrado E, Bosch X, Rodríguez JJ et al. Tratamiento del infarto agudo de miocardio en España en el año 2000. El estudio PRIAMHO II. Rev Esp Cardiol 2003; 56: 1165-1173.

10. Hernández-García J, Medina-Osuna A, GarzónSigLER R. Manejo extrahospitalario de los pacientes atendidos por dolor torácico en tres dispositivos móviles de cuidados críticos y urgencias. Emergencias 2013; 25: 13-22. 
11. Alonso J, Bueno H, Bardají A, García-Moll X, BADía X, LAYOLA M et al. Influencia del sexo en la mortalidad y el manejo del síndrome coronario agudo en España. Rev Esp Cardiol Supl. 2008; 8: 8D-22D.

12. Dziewierz A, Siudak Z, Raokowski T, Dubiel JS, Dudek D. Age-related differences in treatment strategies and clinical outcomes in unselected cohort of patients with ST-segment elevation myocardial infarction transferred for primary angioplasty. J Thromb Thrombol 2012; 43: 214-221.

13. Riesgo A, Bragulat E, López-Barbeito B, SÁnchez M, Miró O. Aproximación diagnóstica al do- lor torácico en urgencias: ¿existen diferencias entre hombres y mujeres? Emergencias 2008; 20: 399-404.

14. Peiró MA, Sierra C, Zamorano M, Muñoz A, AlMELA M. Análisis de las causas de la demora prehospitalaria en el IAM en las mujeres. Enfermería en Cardiología. 2008; 42: 29-33.

15. Hellström K, Brulin C, NäSlund U, Eliasson M. Longer pre-hospital delay in first myocardial infarction among patients with diabetes: an analysis of 4266 patients in the Northern Sweden MONICA Study. Bmc Cardiovasc Disord 2013; 6: 1-7. 